\title{
Des bureaux d'hygiène municipaux aux unités sanitaires. Le Conseil d'hygiène de la province de Québec et la structuration d'un système de santé publique, 1886-1926
}

\author{
Denis Goulet, Gilles Lemire et Denis Gauvreau
}

Volume 49, numéro 4, printemps 1996

URI : https://id.erudit.org/iderudit/305462ar

DOI : https://doi.org/10.7202/305462ar

Aller au sommaire du numéro

Éditeur(s)

Institut d'histoire de l'Amérique française

ISSN

0035-2357 (imprimé)

1492-1383 (numérique)

Découvrir la revue

Citer cet article

Goulet, D., Lemire, G. \& Gauvreau, D. (1996). Des bureaux d'hygiène municipaux aux unités sanitaires. Le Conseil d'hygiène de la province de Québec et la structuration d'un système de santé publique, 1886-1926. Revue d'histoire de l'Amérique française, 49(4), 491-520.

https://doi.org/10.7202/305462ar

\section{Résumé de l'article}

Cet article rend compte des difficultés d'application de certaines politiques sanitaires et préventives rencontrées par le Conseil d'hygiène de la province de Québec lors du processus de structuration du premier système de santé publique permanent couvrant l'ensemble du territoire québécois durant les deux premières décennies du $\mathrm{XX}^{\mathrm{e}}$ siècle. Analysant les avenues privilégiées par le Conseil d'hygiène pour favoriser la prévention des maladies infectieuses, les auteurs mettent en évidence le rôle donné aux municipalités de 1886 à 1926 dans la création d'un système de santé publique. L'article met aussi en évidence les résistances de certains membres des administrations municipales et de la profession médicale, particulièrement dans les petites municipalités, face à certaines mesures imposées et/ou suggérées par le Conseil d'hygiène.
Tous droits réservés @ Institut d'histoire de l'Amérique française, 1996

Ce document est protégé par la loi sur le droit d'auteur. L'utilisation des services d’Érudit (y compris la reproduction) est assujettie à sa politique d'utilisation que vous pouvez consulter en ligne.

https://apropos.erudit.org/fr/usagers/politique-dutilisation/ 


\title{
DES BUREAUX D'HYGIÈNE MUNICIPAUX AUX UNITÉS SANITAIRES. LE CONSEIL D'HYGIÈNE DE LA PROVINCE DE QUÉBEC ET LA STRUCTURATION D'UN SYSTÈME DE SANTÉ PUBLIQUE 1886-1926'
}

\author{
DENIS GOULET \\ Centre de recherche du Centre hospitalier Côte-des-Neiges \\ Département des sciences humaines \\ Université de Sherbrooke \\ GILLES LEMIRE \\ DENIS GAUVREAU \\ Centre de recherche du Centre hospitalier Côte-des-Neiges
}

\section{RÉSUMÉ}

Cet article rend compte des difficultés d'application de certaines politiques sanitaires et préventives rencontrées par le Conseil d'hygiène de la province de Québec lors du processus de structuration du premier système de santé publique permanent couvrant l'ensemble du territoire québécois durant les deux premières décennies du $\mathrm{XX}^{\mathrm{e}}$ siècle. Analysant les avenues privilégiées par le Conseil d'hygiène pour favoriser la prévention des maladies infectieuses, les auteurs mettent en évidence le rôle donné aux municipalités de 1886 à 1926 dans la création d'un système de santé publique. L'article met aussi en évidence les résistances de certains membres des administrations municipales et de la profession médicale, particulièrement dans les petites municipalités, face à certaines mesures imposées et/ou suggérées par le Conseil d'hygiène.

\section{ABSTRACT}

This article shows how, during the decade 1900 and 1910 when the first permanent provincial public health system was structured, some sanitary and preventive policies made by the Board of Health of the Province of Quebec encountered practical problems. Analysing the avenues taken by the Provincial Board

1. Nous remercions le Fonds de la Recherche en Santé du Québec pour son aide financière. Nous remercions également Pierre Lanthier et Jean-Pierre Thouez pour leurs judicieux commentaires.

RHAF, vol. 49, no 4, printemps 1996 
of Health to support the prevention of infectious diseases, the authors underline the role played by the municipalities between 1886 and 1926 in the creation of a public health system. This article emphasizes also the opposition of some members of the municipal administration and medical profession, particularly in small towns, against some measures that were enforced or advised by the Board of Health.

Depuis quelques années, les études sur l'émergence de l'hygiène publique durant les premières décennies du $\mathrm{XX}^{\mathrm{e}}$ siècle ont eu tendance à mettre en évidence le rôle joué par la profession médicale dans la mise en place des mesures sanitaires en territoire québécois tout en soulignant l'inertie des pouvoirs publics qui auraient considéré, à l'instar des établissements hospitaliers, que cette matière relevait en grande partie du secteur privé. Une telle approche incline généralement à affirmer que le Québec aurait subi un retard plus ou moins important dans la mise en place d'un système sociosanitaire permanent. Les visées réformistes de la profession médicale, tant dans le domaine hospitalier que dans le domaine sociosanitaire, relèveraient par ailleurs essentiellement de stratégies visant à protéger leurs intérêts professionnels et à accroître leur pouvoir au sein d'une société en voie de médicalisation laissant par là supposer l'action concertée d'un corps homogène unifié. De telles perspectives, sans être dénuées d'une certaine part de vérité, méritent quelques retouches importantes. Des travaux récents sur l'histoire hospitalière ont montré le rôle important joué par l'État dès les débuts du $\mathrm{XX}^{\mathrm{e}}$ siècle dans la structuration d'un système hospitalier québécois de même qu'ils ont mis en évidence les positions ambiguës d'une profession médicale peu homogène dont les membres ne partagent pas tous le même intérêt pour les nouvelles pratiques diagnostiques, thérapeutiques et sanitaires.

L'analyse très fouillée de François Guérard sur le développement de la santé publique à Trois-Rivières et à Shawinigan s'inscrit dans un renouveau historiographique qui nuance ou modifie les perceptions antérieures ${ }^{2}$. Guérard montre pertinemment l'implication croissante de l'État et de la grande entreprise dès les premières décennies du $\mathrm{XX}^{\mathrm{e}}$ siècle dans la constitution d'une médecine préventive, souligne l'opposition de certains médecins à la déclaration obligatoire des maladies infectieuses et met en évidence la collaboration du clergé vis-à-vis certaines transformations sociosanitaires. Guérard indique

2. F. Guérard, La santé publique dans deux villes du Québec de 1887 à 1939: TroisRivières et Shawinigan, thèse de doctorat (histoire), Université du Québec à Montréal, 1993. Sur l'histoire hospitalière, voir les récents travaux de F. Guérard, de F. Rousseau et de D. Goulet, F. Hudon, O. Keel. 
par ailleurs le rôle de soutien joué par l'élite locale - notamment la petite-bourgeoisie conservatrice et clérico-nationaliste - de TroisRivières aux efforts du Conseil d'hygiène de la province de Québec $(\mathrm{CHPQ})^{3}$. Une telle collaboration, qui connaît certes d'importantes fluctuations, est aussi effective pour la municipalité de Sherbrooke. Ces exemples reflètent-ils une disposition généralement positive des élites municipales des villes moyennes de la province envers les mesures prônées par les autorités du CHPQ? Il faudra d'autres études régionales de ce type pour préciser cette question. Certains éléments présentés dans notre étude indiquent néanmoins que les résistances furent importantes au sein des petites municipalités. Mais le but de cet article n'est pas de reprendre les éléments d'analyse de Guérard, avec lesquels nous sommes généralement en accord, concernant les facteurs économiques et idéologiques, les rapports sociaux et le rôle des élites locales dans la structuration d'un système de santé publique.

Nous voulons rendre compte de façon globale de la difficile transition d'un système ponctuel et temporaire d'intervention des pouvoirs publics en matière de santé publique vers un système centralisé permanent de normalisation, d'imposition et de spécialisation des pratiques préventives. L'analyse des prémisses d'une structuration de la santé publique met en évidence l'implication des élites médicales et des autorités gouvernementales qui désirent suivre adéquatement, et sans décalage sensible avec les autres pays occidentaux, les nouveaux préceptes préventifs légitimés et fondés sur le développement de la bactériologie médicale, à savoir la mise en place d'une chaîne de déclaration des maladies infectieuses, l'amélioration des conditions sanitaires et l'intervention éducative en matière d'hygiène domestique. Cet article ne prétend nullement rendre compte de façon exhaustive des multiples facettes de cette structuration mais en souligne les principaux éléments: renforcement de la législation sanitaire, complexité des rapports entre les pouvoirs municipaux et provinciaux, difficultés d'application et d'uniformisation des règles d'hygiène, hétérogénéité des attitudes médicales, collaboration du clergé en matière de déclaration des maladies infectieuses, adhésion des membres du CHPQ aux options réformistes instaurées dans les principaux pays occidentaux. Plus globalement, notre étude se veut une contribution à la généalogie de la médecine préventive en territoire québécois.

3. Pour la commodité du lecteur nous emploierons le sigle CHPQ pour désigner la Commission provinciale d'hygiène de la Province de Québec (1886-1888); le Conseil d'hygiène de la Province de Québec (1888-1914); le Conseil supérieur d'hygiène de la Province de Québec (1915-1922) et le Service provincial d'hygiène de la Province de Québec (1923-1926). 


\section{1 - LE CONSEIL D'HYGIÈNE DE LA PROVINCE DE QUÉBEC ET LA STRUCTURATION D'UN SYSTÈME DE PRÉVENTION DES MALADIES INFECTIEUSES}

Jusqu'à la décennie 1880 , les interventions des autorités gouvernementales canadiennes et québécoises en matière de santé publique se limitaient généralement à l'imposition de mesures de quarantaine et de vaccination, à l'érection d'hôpitaux pour contagieux et à l'octroi de fonds pour les soins aux marins, aux aliénés, aux infirmes et aux orphelins. Certaines mesures d'urgence, surtout axées sur les centres urbains, étaient adoptées de façon ponctuelle et temporaire pour répondre à l'éclosion périodique de grandes épidémies de choléra, de typhus ou de variole.

Sans noircir exagérément le tableau des conditions sanitaires jusqu'à la fin du XIX ${ }^{\mathrm{e}}$ siècle, on peut dire qu'elles étaient généralement médiocres: la qualité de l'eau potable et du lait était parfois mauvaise, l'ébouage n'était pas encore une mesure uniforme et répandue, les rares systèmes d'adduction d'eau étaient souvent mal entretenus et les systèmes d'égout et d'écoulement des eaux demeuraient une technique sanitaire marginale. Enfin, le taux de mortalité infantile était généralement élevé et les petites épidémies demeuraient fréquentes ${ }^{4}$.

Il y eut bien l'amorce d'une prise en charge des conditions sanitaires par les autorités municipales de Montréal et de Québec au tournant des années $1870^{5}$, mais c'est à partir de la décennie 1880 que furent véritablement établies des structures permanentes de contrôle de la santé publique pour l'ensemble du territoire québécois. Plusieurs raisons ont présidé à la nécessité de structurer les mesures d'hygiène publique: poussée démographique, développement industriel, accroissement des accidents de travail et, par conséquent, des besoins de soins rapides et adéquats, connaissance accrue des causes et des vecteurs des maladies infectieuses grâce aux découvertes bactériologiques $^{6}$, nécessité de prévenir de façon plus rigoureuse la circulation

4. Les conditions sanitaires étaient-elles aussi mauvaises que le laisse entendre généralement l'historiographie? Quant aux taux de mortalité infantile, ils apparaissent certes très élevés, mais une étude serrée des causes de cette mortalité pour l'ensemble du Québec permettrait de préciser cette question. Sur la mortalité infantile à Montréal au XIX ${ }^{\mathrm{e}}$ siècle, voir les travaux de $\mathrm{S}$. Olson et $\mathrm{P}$. Thorton.

5. Voir M. Farley, O. Keel et C. Limoges, «Les commencements de l'administration montréalaise de la santé publique (1865-1885)», Scientia Canadensis, 20 (1982): 24-46 et 21 (1982): 85-109.

6. Sur les thèses contagionnistes et infectionnistes qui ont prévalu en Europe et en Amérique du Nord jusqu'à la fin du $\mathrm{XIX}^{\mathrm{e}}$ siècle, lesquelles impliquaient une perception environnementale et, dans une moindre mesure, sociale de la santé publique - néo-hippocratisme, miasmes, contages - et la transition importante vers la théorie des germes issue des travaux de 
des malades contagieux à la suite de l'accroissement des échanges marchands, interventionnisme plus vigoureux de la profession médicale, etc.

C'est en réponse à une situation de crise sociale, comme cela est fréquent dans l'histoire de la santé au Québec comme ailleurs ${ }^{7}$, qu'est instituée légalement la première structure provinciale permanente de santé publique. La promulgation de la loi de santé publique qui entraîne la fondation de la Commission provinciale d'hygiène de Québec en 1886, dénommé officiellement Conseil d'hygiène de la province de Québec (CHPQ) en 1888, constituait en quelque sorte une réponse à l'importante épidémie de variole qui affecta le Québec en 1885. Première structure provinciale permanente de santé publique au Québec, le CHPQ obtenait le mandat de répondre aux besoins d'organisation sanitaire de la province ${ }^{8}$, d'encourager la formation de bureaux sanitaires municipaux, de surveiller l'application des lois d'hygiène, de favoriser la prévention des maladies infectieuses et d'assurer la distribution des vaccins et des sérums. Le CHPQ se dotera d'un service d'inspection générale, d'un service des maladies contagieuses, d'un service de génie sanitaire, d'un service de statistique et d'un laboratoire de chimie et de bactériologie.

Orientant surtout leurs efforts vers la prévention des maladies infectieuses, les membres du CHPQ souhaitaient constituer une médecine préventive largement interventionniste. Un tel projet impliquait deux niveaux d'intervention: a) la recherche d'une collaboration

Pasteur, de Koch, et al., voir F. Delaporte, Le savoir de la maladie. Essai sur le choléra de 1832 à Paris (Paris, Presses universitaires de France, 1990); D. Goulet, «Entre les miasmes et les germes. L'impact de la bactériologie sur la pratique médicale en territoire canadien (18701930)», Historia, Ciências, Saude - Manguinhos, 1,2 (1995): 20-38; D. Goulet et O. Keel, «Généalogie des représentations et attitudes face aux épidémies au Québec depuis le XIX ${ }^{\circ}$ siècle», Anthropologie et Sociétés, 15,2-3 (1991): 205-228; P. Keating et O. Keel, «Autour du Journal de médecine de Québec/Quebec Medical Journal (1826-1827): programme scientifique et programme de médicalisation», R. A. Jarrell et A. E. Roose, dir., Problèmes cruciaux de l'histoire de la science, de la technologie et de la médecine au Canada (Thornhill, HSTC Publications, 1983), 101-134.

7. Mentionnons à titre d'exemple qu'à la Nouvelle-Orléans, c'est une épidémie de fièvre jaune «qui servit de catalyseur de l'opinion publique et força les autorités municipales à se préoccuper davantage des problèmes reliés à la santé publique», G. Vandal, «Santé publique et développement urbain: les conditions sanitaires à la Nouvelle-Orléans, 1850-1885», Canadian Journal of History/Annales canadiennes d'histoire, 28 (avril 1993): 6. Sur l'épidémie de variole de 1885, voir M. Bliss, Montréal au temps du grand fléau: l'histoire de l'épidémie de 1885 (Montréal, Libre Expression, 1993).

8. Une loi intitulée Acte pour établir une Commission provinciale d'hygiène, et pour d'autres fins relativement à la santé publique est promulguée en 1886 . Il semble toutefois que cette commission ne deviendra active qu'en 1887. L'année suivante, une nouvelle loi intitulée Acte concernant la santé publique définit les nouvelles responsabilités du Conseil d'hygiène de la Province de Québec. 
étroite des municipalités et des médecins dans l'application de solutions sanitaires réformistes, telles que la mise en place d'un service d'ingénierie sanitaire et la constitution d'un réseau de déclaration des maladies infectieuses; b) la mise sur pied de grandes campagnes de propagande sanitaire axées sur l'hygiène privée et destinées à la population. Si les interventions du CHPQ sont nombreuses en ce qui regarde l'amélioration des infrastructures sanitaires et la promotion de l'hygiène privée, il demeure que, durant les trois premières décennies d'activités du CHPQ, l'un de ses objectifs majeurs sera de mettre sur pied un réseau fiable de déclaration des maladies infectieuses sur l'ensemble du territoire québécois. Or, l'efficacité d'un tel réseau nécessitait la collaboration de chaque individu impliqué dans le processus. Privilégiant la voie de la réglementation, les autorités du CHPQ obtiendront plusieurs amendements à la loi d'hygiène publique ${ }^{9}$ durant les décennies 1900, 1910 et 1920, lesquels auront pour effet de renforcer leur pouvoir d'intervention et de sanction concernant la déclaration des maladies infectieuses et la promotion de meilleures conditions sanitaires.

Dès 1886, l'Acte pour établir une Commission provinciale d'hygiène, et pour d'autres fins relatives à la santé publique, prévoit l'étude de statistiques vitales, la mise en œuvre de perquisitions sanitaires et d'enquêtes sur les causes des maladies, ainsi que l'adoption de mesures de prophylaxie sociale ${ }^{10}$. La loi vise en outre à encourager la formation de commissions locales d'hygiène ${ }^{11}$ et la diffusion d'informations à caractère prophylactique. Elle définit également les devoirs des chefs de famille et des médecins vis-à-vis les autorités de la commission en ce qui regarde la déclaration des malades contagieux ${ }^{12}$. Ceux-ci doivent en effet aviser la «commission locale d'hygiène» ou, à défaut, les officiers d'hygiène ou le maire de la municipalité dès qu'ils sont mis en présence d'une maladie infectieuse ou de toute «autre maladie dangereuse pour la santé publique ${ }^{13}$ ».

Les dispositions de cette loi, votée à la suite de la terrible épidémie de variole de 1885 , constituaient un précédent juridique en ce qui regarde la prévention des maladies infectieuses ${ }^{14}$. Mais le libellé

9. Des amendements à la loi d'hygiène et de nouveaux règlements sont apparus notamment en 1888, 1895, 1898, 1900, 1901, 1902, 1909, 1913, 1915, 1919, 1924, 1926.

10. Statuts de la Province de Québec (SPQ) (1886), chap. 38, art. 5.1, 5.2, 5.3, 5.4.

11. Ibid., art. 5.5 .

12. Ibid., art. 11 et 12 .

13. $S P Q(1886)$, chap. 39 , art. 12 .

14. J. Houle, Santé publique au Québec 1880-1920: évolution de la législation sanitaire du gouvernement provincial étudiée sous l'angle de la "Loi d'hygiène publique de Québec» (texte ronéotypé, 1993). 
n'accordait généralement aux membres de la Commission provinciale d'hygiène qu'un rôle de recommandation à titre d'experts en matière d'hygiène publique $^{15}$. Ceux-ci avaient certes le devoir de favoriser le cumul des statistiques pertinentes en matière de maladies infectieuses, mais ils ne possédaient ni pouvoir réel d'ordonnance ni moyens concrets de sanction contre les négligents ou les contrevenants. $\mathrm{Si}$ cette loi avait l'avantage d'assurer les bases d'une structure provinciale en matière de santé publique, elle s'avérait aux yeux des membres de la commission trop peu contraignante ${ }^{16}$. La loi ne leur accordant qu'un simple pouvoir de «suggérer» et «d'encourager» la formation de bureaux municipaux d'hygiène, les autorités du CHPQ souhaitaient obtenir la possibilité d'ordonner la formation de tels bureaux. À la suite de leurs pressions, l'Assemblée législative modifia en 1888 le statut de cette commission en entérinant la formation du Conseil d'hygiène de la Province de Québec ${ }^{17}$. La nouvelle loi accordait à ses membres un pouvoir d'ordonnance quant à la formation de bureaux municipaux d'hygiène ${ }^{18}$. Cette dernière disposition était jugée essentielle par le CHPQ puisqu'on souhaitait faire de ces bureaux municipaux d'hygiène un maillon essentiel de la structure de déclaration des maladies infectieuses et du dispositif d'hygiène publique projeté pour l'ensemble du Québec. Le CHPQ obtenait par ailleurs le pouvoir de rédiger des règlements relatifs à la prophylaxie des maladies contagieuses ainsi qu'à l'amélioration de la santé publique ${ }^{19}$.

15. Les termes utilisés pour établir les devoirs de la Commission sont révélateurs de l'esprit de cette loi: «étudier spécialement les statistiques», «étudier la manière de prévenir [...], de faire les suggestions [...], d'encourager la formation [...], de donner [...] des avis...», SPQ (1886), chap. 39 , art. 5 .

16. «Le seul pouvoir d'aviser, conféré au CHPQ par la loi de 1886, n'était pas suffisant pour lui permettre d'atteindre le but qu'il poursuit», note rétrospectivement le sécrétaire du CHPQ, le docteur E. Pelletier, Rapport annuel du Conseil d'hygiène de la Province de Québec (RACHPQ) (1895), 13.

17. $S P Q$ (1888), chap. 47.

18. «Le maire ou autre chef de la corporation municipale de chaque localité, après avoir été notifié par écrit par le conseil provincial d'hygiène, doit convoquer une assemblée spéciale du conseil [...] dans laquelle assemblée pas moins de trois personnes résidant dans les limites de leurs juridictions respectives, sont nommées pour former le 'conseil local d'hygiène' pour cette localité», Ibid., art. 6.

19. Ibid., art. 5.3. La loi de 1886 ne contenait aucune disposition lui permettant de réglementer la prévention des maladies infectieuses. Elle stipulait simplement que «la commission a le pouvoir de faire et d'adopter des règles et des règlements pour réglementer sa manière d'agir, et de pourvoir, par ces règlements, à la nomination de comités auxquels elle délègue son autorité et ses pouvoirs pour accomplir la tâche qui lui est assignée», $S P Q$ (1886), chap. 38, art. 7. Or celle de 1888 mentionnait que le CHPQ avait le pouvoir «d'établir des règlements qu'il croit propres à prévenir, autant que possible, ou à mitiger les maladies épidémiques, endémiques et contagieuses, avec pouvoir de révoquer, renouveler et changer ces règlements ou leur en substituer de nouveaux selon qu'il le trouve convenable», Ibid., chap. 47, art. 3 . 


\section{A - Un rôle majeur dévolu aux municipalités}

Le CHPQ, qui ne pouvait certes pas gérer de façon centralisée les conditions sanitaires de plus de 800 municipalités, avait institué, dès sa création, une structure de santé publique largement axée sur la collaboration des autorités municipales. La promulgation et l'application des règlements sanitaires reposeront donc, jusqu'en 1911, sur deux niveaux distincts: une instance provinciale directrice (CHPQ) et une instance municipale exécutrice (bureau d'hygiène local ou conseil municipal). Ce modèle d'intervention basé sur les municipalités était en partie inspiré des locals boards of health qui avaient été établis

\section{ORGANIGRAMME 1}

Organisation sanitaire générale de la Province de Québec

$1886-1926$

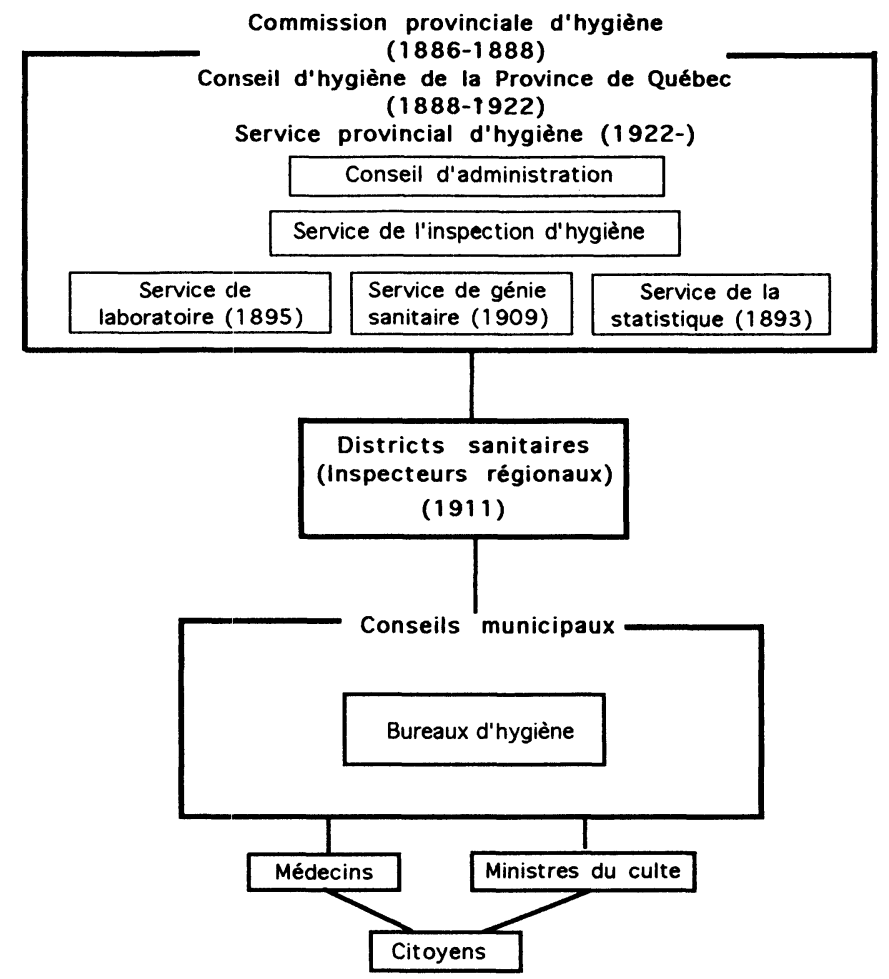

Sources: Rapports annuels du Conseil d'hygiene de la Province de Quebec, 1892 - 1926. 
avec un succès relatif aux États-Unis à la fin du XIX $\mathrm{XIècle}^{20}$. Comme l'indique l'organigramme I, les bureaux locaux d'hygiène servaient de relais entre le médecin ou le citoyen et les autorités sanitaires provinciales.

À partir de 1888, à la suite d'un avis du CHPQ transmis par lettre, les autorités municipales étaient tenues de convoquer une assemblée spéciale du conseil municipal afin de former un bureau d'hygiène municipal composé d'au moins trois membres ${ }^{21}$. Chaque bureau devait désigner des mandataires choisis ou non parmi les membres du conseil municipal chargés de faire appliquer la loi d'hygiène du Québec. Le CHPQ avait aussi recommandé la nomination par le conseil municipal d'un officier sanitaire exécutif. C'est à cet officier que revenait la tâche d'établir un lien de communication efficace entre le CHPQ et le bureau sanitaire municipal. Si le CHPQ recommandait qu'un médecin résidant dans la localité soit désigné à cette fonction, cela n'était pas toujours possible ni même souhaité:

Il n'a pas pu être question de rendre invariablement obligatoire la nomination d'un médecin comme officier exécutif, pour la raison que nombre de municipalités n'ont pas de médecin résident dans leurs limites et que, dans d'autres cas, les médecins qui y résident se soucient malheureusement trop peu de l'hygiène. [...] Si une municipalité n'a dans ses limites, qu'un médecin qui ne déclare jamais ses cas de maladies contagieuses, ou qui a été pris à faire des simulacres de désinfection, elle doit le tenir en dehors de son organisation sanitaire $[\ldots]^{22}$.

Les médecins éligibles au titre d'officier sanitaire n'étaient pas toujours faciles à recruter ${ }^{23}$. Le salaire octroyé à l'officier exécutif était plutôt modeste, inférieur parfois au salaire du chef de police ou du chef des pompiers. Du reste, plusieurs médecins, nous le verrons, ne partageaient pas l'enthousiasme des membres du CHPQ vis-à-vis le

20. Tout comme dans le Massachusetts, le Michigan Board of Health avait obtenu en 1877 une loi «requiring each township to appoint a health officer», J. Duffy, The Sanitarians. A History of American Public Health (Urbana, University of Illinois Press, 1990), 151.

21. «Le maire ou autre chef de la corporation municipale de chaque localité, après avoir été notifié par écrit par le conseil provincial d'hygiène, doit convoquer une assemblée spéciale du conseil ou autre corporation municipale de telle localité qu'il préside, dans laquelle assemblée pas moins de trois personnes résidant dans les limites de leurs juridictions respectives, sont nommées pour former le 'conseil local d'hygiène' pour cette localité», $S P Q$ (1888), chap. 47, art. 6 .

22. $R A C H P Q(1908), 23$.

23. Le sécrétaire du CHPQ mentionne avec irritation en 1908 «que le salaire du médecin municipal y est inférieur à celui payé aux autres chefs de départements, voire même au salaire du chef de police et du chef des pompiers», Ibid. 
contrôle sanitaire. Il n'était donc pas rare que cette tâche soit plutôt confiée au sécrétaire-trésorier ${ }^{24}$ ou, dans le pire des cas, à ceux «que l'hygiène laisse indifférent et qui s'étaient le plus opposés à la formation de tels bureaux ${ }^{25}$ ». En vertu d'une faille de cette loi qui ne précisait pas la durée élective de ces bureaux, certaines municipalités réticentes aux mesures d'hygiène prenaient la liberté de dissoudre leur bureau d'hygiène peu de temps après sa formation laissant au conseil municipal l'application des mesures sanitaires ${ }^{26}$.

En 1891, moins de la moitié des municipalités s'étaient conformées aux ordonnances du conseil. Même si l'autorité du CHPQ avait été quelque peu renforcée par la loi de 1888, l'application des règlements d'hygiène au tournant de la décennie 1890 se heurtait encore souvent à l'inertie, voire à l'opposition farouche de certaines municipalités. Cependant, l'épidémie de variole qui affecta la province en 1891 catalysa les efforts du CHPQ et sensibilisa de nombreuses municipalités à l'efficacité d'un bureau d'hygiène susceptible d'atténuer de telles épidémies. En outre, le CHPQ, par l'intermédiaire de son président, le docteur E.-P. Lachapelle, et de son trésorier, le docteur E. Pelletier, désirait accroître leur pouvoir de contrainte face aux autorités récalcitrantes. Les amendements survenus à la loi d'hygiène publique en 1890 et 1894 répondront en partie aux demandes du conseil ${ }^{27}$. Le Conseil d'hygiène avait obtenu, outre l'imposition d'amende en cas de refus d'exécution d'une ordonnance du conseil, «le pouvoir de procéder, aux frais de la municipalité en faute, à l'exécution des règlements enfreints ${ }^{28}$ ». En 1895,839 des 876 municipalités de la province avaient établi leur bureau local d'hygiène ${ }^{29}$.

De nombreuses tâches d'hygiène publique, sur la recommandation du CHPQ, furent confiées à ces bureaux locaux: limiter l'extension des maladies contagieuses; supprimer les nuisances sanitaires; contrôler le drainage des maisons, la construction et l'entretien des fosses d'aisances; surveiller l'élevage d'animaux ainsi que la construction et

24. En l'absence d'officier exécutif pour ce bureau, le secrétaire-trésorier de la municipalité devient ex-officio l'officier sanitaire exécutif. Et en l'absence de candidats pour ce bureau, ce sont des membres du conseil municipal qui y sont désignés, Ibid. (1896), 8.

25. Ibid. (1895), 22.

26. Pour corriger cette lacune, un amendement à la loi survenue en 1894 stipule qu'aucun bureau d'hygiène municipal ne pourra être ainsi aboli. Cet amendement stipule aussi que les conseils municipaux peuvent «faire des règlements pour la régie interne de son bureau d'hygiène», $S P Q$ (1894), chap. 31, amendement de l'art. 3063.

27. Voir à ce propos ibid. (1890), chap. 27, art. 3066s.

28. Ibid. (1895), 14.

29. Ibid., 21. 
l'entretien des abattoirs dans les municipalités; contrôler les dépôts d'ordures ou de matières nuisibles; inspecter les viandes et le lait; éviter la contamination des cours d'eau; veiller à la qualité de l'eau potable et de la glace; enfin, de façon plus générale, s'efforcer de promouvoir la propreté. À la suite de la loi d'hygiène publique de 1895, les conseils municipaux devaient transmettre au Conseil d'hygiène un rapport annuel sur leurs opérations sanitaires.

Outre l'installation d'équipements relevant de l'ingénierie sanitaire, certaines activités préventives impliquaient, en priorité, la mise en œuvre de campagnes de propagande sanitaire et l'imposition de mesures visant à standardiser la déclaration de tous les cas de maladies infectieuses. À ce propos, le CHPQ élaborera durant les premières décennies du $\mathrm{XX}^{\mathrm{e}}$ siècle des stratégies plus ou moins contraignantes afin d'obtenir une précision accrue dans la quantification et la localisation des maladies infectieuses.

\section{B - Une première chaîne de déclaration des maladies contagieuses}

La Loi d'hygiène publique de 1888 obligeait les familles et les médecins, sous peine d'une amende n'excédant pas vingt piastres, à déclarer aux autorités municipales tous les cas de maladie infectieuse ${ }^{30}$. Le malade et sa famille constituaient les premiers maillons de cette chaîne de déclaration. Toute famille informée de la présence d'un malade contagieux en son sein se voyait contrainte d'aviser immédiatement l'officier exécutif du bureau municipal d'hygiène ou à défaut, le secrétaire-trésorier de leur municipalité. Aussi, la loi d'hygiène obligeait tout médecin appelé auprès des malades contagieux à en informer la municipalité et à déclarer le lieu de résidence des personnes atteintes. Les municipalités avaient la responsabilité de faire parvenir mensuellement au CHPQ le registre des maladies contagieuses déclarées sur son territoire ${ }^{31}$.

À partir de 1901, un amendement à la loi obligea «les municipalités à déclarer le premier cas d'une maladie contagieuse par lettre recommandée déposée au bureau de poste dans les vingt-quatre heures après en avoir obtenu connaissance [...] et de lui fournir, tant que la maladie existe, tous les huit jours, ou plus souvent si le conseil d'hygiène le demande, un état indiquant le nombre de nouveaux cas constatés, ainsi que le chiffre de ceux qui sont morts, guéris ou encore malades ${ }^{32}$ ». Même si le CHPQ disposait de certains recours pénaux

30. $S P Q(1888)$, chap. 47 , art. 12 et 13.

31. RACHPQ (1901), 10.

32. $S P Q(1901)$, chap. 19 , art. 53. 
ORGANIGRAMME II

Déclaration des maladies infectieuses

1888-1926

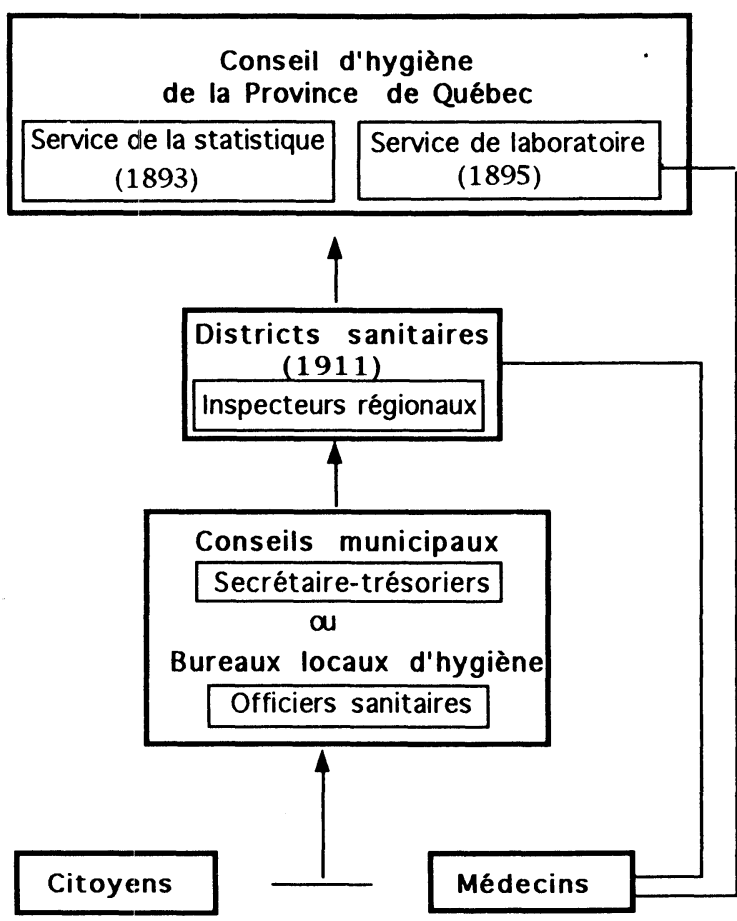

Sources: Rapports annuels du Conseil d'hygiène de la Province de Québec, 1911 - 1926.

auprès des intervenants, notamment l'imposition d'amende, cette chaîne de déclaration, illustrée dans l'organigramme II, reposait sur la collaboration volontaire de chaque partie. En effet, comme le souligne le secrétaire du Conseil d'hygiène, peu de poursuites furent intentées contre les contrevenants.

Outre la déclaration des cas de maladies infectieuses, le Conseil avait aussi le mandat de recueillir les déclarations de décès pour l'ensemble de la province de Québec. En 1893, après quatre ans d'efforts et de fortes résistances de la part de ceux qui y voyaient une loi conduisant à l'enregistrement civil, le CHPQ obtint de la 
Législature l'adoption d'une «loi de la statistique ${ }^{33} »$, complément nécessaire à toute loi d'hygiène publique. Les ministres du culte, selon les dispositions du Code civil relativement à la tenue des registres par le clergé, agissaient à titre de préposés à l'enregistrement des actes de l'état civil et devaient faire parvenir mensuellement au CHPQ, et par voie postale, les certificats sur lesquels était inscrite la cause du décès ${ }^{34}$. En principe, c'est aux médecins que revenaient la responsabilité d'attester la cause du décès, laquelle devait être notifiée sur le certificat $^{35}$. Toutefois, en l'absence du médecin, le ministre du culte ou «deux personnes dignes de foi» étaient autorisés à établir, «au meilleur de leur connaissance la cause du décès ${ }^{36}{ } »$. Ceci ne manqua pas de susciter quelques imprécisions diagnostiques et, comme le souligne le préposé aux statistiques du CHPQ, le docteur Paul E. Prévost, de nuire «à la connaissance des vraies causes de mortalitée ${ }^{37}$ ». À partir de 1909, les coroners et les juges de paix étaient aussi mandatés, en l'absence du médecin, pour établir les certificats de décès.

La chaîne de déclaration, telle qu'illustrée par l'organigramme III impliquait une collaboration étroite entre les médecins et les ministres du culte. L'efficacité d'une telle procédure de déclaration, section essentielle en matière d'hygiène publique ${ }^{38}$, dépendait évidemment de l'exhaustivité des rapports de décès et de la régularité des envois au CHPQ. À cet égard, le médecin constituait le maillon essentiel de cette chaîne de déclaration puisqu'il était le seul garant de la précision du diagnostic. Or il semble que de nombreux certificats de décès adressés au CHPQ étaient rédigés par les ministres du culte seuls ce qui constituait «une mesure déplorable et tout à fait contraire à l'esprit de la $10^{39}{ }^{39}$. Le compilateur du bureau de la statistique considérait, non sans raison, que seuls les médecins étaient aptes à établir la cause du décès ${ }^{40}$. En conséquence, les certificats de décès délivrés au conseil,

33. Ibid. (1893), «Loi concernant la compilation des statistiques relatives au mouvement de la population», chap. 29.

34. De par cette loi, les ministres du culte devaient aussi, à la fin de chaque année, transmettre au Conseil d'hygiène le nombre total des mariages et des naissances qui avaient eu lieu pendant l'année, dans leur juridiction, $R A C H P Q$ (1895), 17. Traditionnellement, les curés de paroisses et leurs assistants avaient la charge d'établir le registre des décès. Voir $S P Q$ (1893), chap. 29 , art. $3059 \mathrm{e}$.

35. Ibid.

36. Ibid.

37. RACHPQ (1898), 72. La loi de statistique stipulait aussi que les certificats devaient être détruits immédiatement après la compilation des statistiques, $S P Q$ (1893), chap. 29, art. 3059e.

38. «[...] dans les questions du domaine de la démographie, la maladie qui a été la cause du décès a une importance considérable, et doit être réellement considérée comme un facteur fondamental qu'il ne faut pas laisser de côté», $R A C H P Q$ (1897), 73.

39. Ibid.

40. Ibid. 


\section{ORGANIGRAMME III}

Déclaration des décès $1893-1926$

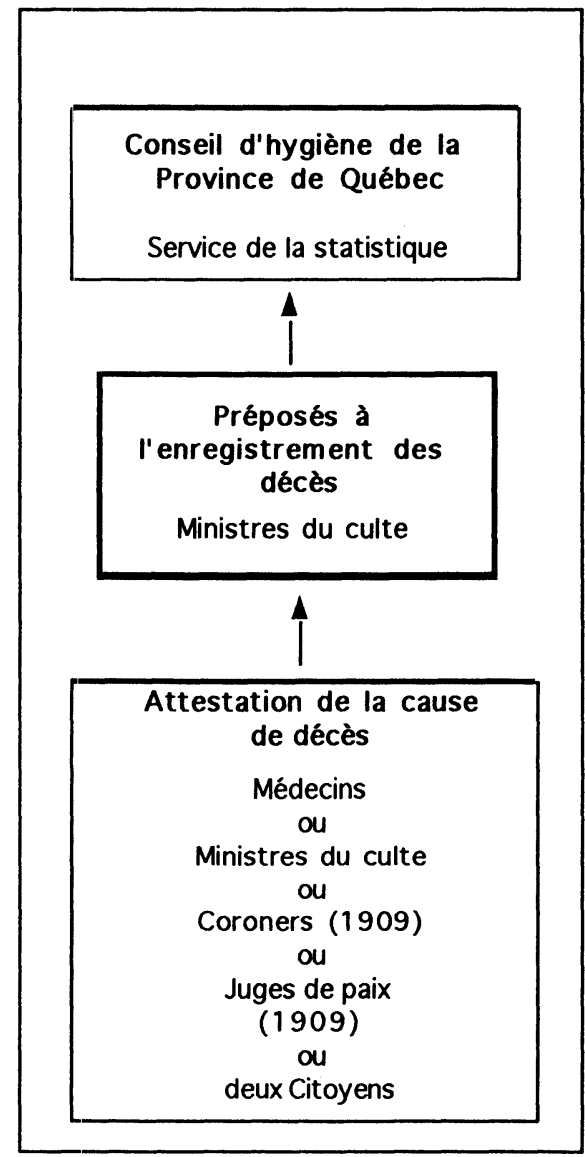

Sources: Rapports annuels du Conseil d'hygiène de la Province de Québec, 1892 - 1926.

selon qu'ils étaient ou non attestés par le médecin, n'avaient pas la même valeur statistique même si le compilateur reconnaît que dans bien des cas «la maladie inscrite comme cause de décès est bien celle 
diagnostiquée par le médecin». Autre problème: certains prêtres omettaient de faire parvenir au conseil les certificats de décès rédigés par le médecin traitant ou, en son absence, par deux témoins, avant de procéder à l'inhumation, tel que la loi le prescrivait ${ }^{41}$.

Il faut toutefois souligner que les négligences de ce genre tendront à diminuer à partir de la décennie 1900. En général, la structure de déclaration des décès fonctionnera avec une relative efficacité et les autorités cléricales collaboreront étroitement avec le CHPQ. Celui-ci pouvait, en effet, compter sur l'appui des évêques qui ne manquaient pas de semoncer les prêtres négligents ${ }^{42}$. De plus, le conseil d'hygiène veillait à ce que ces compilations statistiques jugées essentielles «pour l'avenir de [la] race [soient] marquées au coin (sic) de la plus rigoureuse exactitude ${ }^{43}{ }^{\prime}$. Des moyens de pression auprès des négligents seront donc mis en œuvre pour atteindre cet objectif. En 1909, le compilateur de la statistique note, avec satisfaction, que les ministres du culte font preuve d'une plus grande ponctualité dans l'envoi de leur rapport mensuel ${ }^{44}$. Durant les décennies 1910 et 1920, les rapports seront acheminés au conseil avec de plus en plus de régularité. Certaines hausses rapides des statistiques de décès sont attribuables en partie à cette amélioration ${ }^{45}$. La tendance vers une plus grande fiabilité des données épidémiologiques concernant les décès devenait irréversible.

En 1919, le compilateur de la statistique constate avec satisfaction qu'il est «en mesure de pouvoir publier des chiffres suffisamment complets sur les statistiques vitales et mortuaires de cette province ${ }^{46}$ ". Durant la décennie 1920, les rapports statistiques étant jugés satisfaisants, le conseil adressera à quelques reprises ses félicitations aux ministres du culte ${ }^{47}$. Ceux-ci, à la suite d'une refonte de la loi des

41. Voir à ce propos: $R A C H P Q$ (1900), 57; (1902), 82-83; (1903), 68; (1907, 98; Rapport annuel du Conseil supérieur d'hygiène de la Province de Québec (RACSHPQ) (1916), 140.

42. «Nous regrettons de constater que quelques préposés aux registres, malgré nos démarches répétées et celles de l'autorité diocésaine, qui nous seconde invariablement, persistent à ne pas vouloir nous envoyer les certificats de décès conformément aux prescriptions de la loi», ibid. (1900), 56.

43. Ibid. (1909), 79.

44. Ibid. (1908), 83.

45. «[...] la mortalité de l'année 1908 l'emporte de 5240 sur celle de l'année précédente. Une partie de cette augmentation est sans doute attribuable à la plus grande ponctualité de MM. les Ministres du culte, à nous faire parvenir leur rapport mensuel», ibid. Pour l'année 1913, les statistiques indiquent 3220 décès de plus que l'année précédente.

46. RACSHPQ (1919), 164.

47. «Nous terminerons en disant que les chiffres statistiques que nous donnons sur la mortalité de cette province sont à peu près complets. Nous en félicitons bien sincèrement les préposés à l'enregistrement des actes de l'État Civil qui sont fidèles à nous envoyer leur rapport au commencement de chaque mois», ibid. (1920), 150. 
statistiques démographiques promulguée en 1926, seront officiellement désignés «collecteurs des statistiques démographiques ${ }^{48}$ ». Cette décision fut évidemment bien accueillie par les autorités religieuses.

Avantage non négligeable, la fiabilité des compilations de décès causés par maladies infectieuses, à partir de la décennie 1910, permettait aux autorités du conseil de «contrôler, dans une certaine mesure, si la déclaration des maladies contagieuses est régulièrement faite aux autorités sanitaires municipales ${ }^{49}{ }$. Les statistiques concernant la déclaration des maladies infectieuses étaient jaugées à l'aune des déclarations de décès. Or une telle évaluation comparative n'était guère flatteuse pour les bureaux municipaux d'hygiène. Il est indéniable que le personnel clérical s'acquittait de sa responsabilité statistique avec plus de célérité et d'assiduité que les médecins et les officiers municipaux. Or, nous le verrons, les défaillances dans la chaîne de déclaration des maladies infectieuses constituent un indice important de la précarité des structures sanitaires municipales.

\section{C - Des municipalités rurales récalcitrantes}

Si les mesures d'hygiène publique proposées par le CHPQ s'avéraient impératives, elles ne seront pourtant pas toujours appliquées par les autorités municipales. En 1895, le secrétaire du CHPQ reconnaît certes que «le personnel des bureaux locaux paraît avoir été choisi avec soin dans la plupart des municipalités» et qu'on a fait «en général, de ces bureaux une organisation sérieuse», mais il souligne en revanche que certaines municipalités ne comprennent «pas encore suffisamment l'importance d'un bureau local d'hygiène», et que plusieurs ont formé un bureau pour se conformer à la loi mais sont restées indifférentes à l'hygiène ${ }^{50}$. Quatorze ans plus tard, le propos est plus sévère: «L'inertie de nombre de nos municipalités, en matière d'hygiène, est déplorable; on n'y fait absolument rien pour empêcher la propagation de maladies contagieuses et pour supprimer les nuisances ${ }^{51} . »$

Ce laxisme des autorités locales provient en partie du fait qu'elles envisageaient le rôle de ces bureaux comme étant essentiellement une mesure temporaire d'urgence destinée à enrayer la propagation d'une épidémie. Certaines représentations traditionnelles des causes et des vecteurs des maladies infectieuses rendaient sceptiques les autorités

48. Rapport annuel du Service provincial d'hygiène de la Province de Québec (RASPHPQ) (1926), 10.

49. RACSHPQ (1916), 8 .

50. Ibid., 22-23.

51. RACHPQ (1909), 52-53. 
municipales quant à l'efficacité des mesures prônées par les hygiénistes. De fait, il n'était pas rare que certaines mesures prophylactiques découlant des recherches bactériologiques se heurtaient à des représentations qui rendaient certaines causes climatiques responsables des maladies contagieuses au détriment, par exemple, du vecteur hydrique ${ }^{52}$.

Les membres du CHPQ désiraient modifier cette situation qui avait prévalu tout au long du $\mathrm{XIX}^{\mathrm{e}}$ siècle. Il fallait imposer l'idée nouvelle que la lutte contre les maladies infectieuses - élément central de toute mesure d'hygiène publique - doit être désormais effectuée, non de façon ponctuelle lors de l'éclosion d'une épidémie, comme cela était monnaie courante au $\mathrm{XIX}^{\mathrm{e}}$ siècle, mais quotidiennement par l'imposition et le respect rigoureux de mesures préventives découlant en partie des nouveaux acquis de la science bactériologique. Il est hors de propos de présenter ici l'ensemble des réformes proposées par le CHPQ, réformes qui touchaient à tous les secteurs de la vie municipale ${ }^{53}$. Mentionnons simplement, à titre d'exemple, certaines recommandations du CHPQ auprès des municipalités afin qu'elles établissent «un plan de développement résidentiel par lequel on pourrait établir l'orientation des rues, leur largeur, les réserves pour parcs publics, etc. ${ }^{54}$ ». Une telle mesure visait à modifier la «construction d'habitations ouvrières [...] entièrement laissée entre les mains des spéculateurs [...] qui ont d'autres intérêts que l'hygiène des habitations ${ }^{55}$ ».

Il est certain que les contraintes économiques sont en partie responsables des résistances des autorités municipales, particulièrement en milieu rural, vis-à-vis certains projets d'ingénierie sanitaire.

52. Howard Kremer mentionne que dans les années 1880 le Congrès américain a abandonné son support au Bureau national de santé créé en 1879 et ajoute que «the failure of this early program can be attributed in great part to medical ignorance concerning the true etiology of disease», H. D. Kramer, «The Germ Theory and the Early Public Health Program in the United States", Bulletin of the History of Medicine, 12 (1948): 233. Voir aussi A.-I. Marcus, «The Strange Career of Municipal Health Initiatives: Cincinnati and City Government in the Early Nineteenth Century», Journal of Urban History, 7,1 (1980): 3-29; M.-V. Melosi, «'Out of Sight, Out of Mind': the Environmental and Disposal of Municipal Refuse, 1860-1920», The Historian, 35 (1973): 621-640.

53. Sur l'application des politiques d'hygiène dans les régions, voir pour la Mauricie: F. Guérard, op. cit.; pour les Cantons de l'est: J. Pomerleau, Le problème de l'application locale des politiques d'hygiène du Gouvernement québécois avant 1920, avec référence aux comtés de Brome et Missisquoi, mémoire de maîtrise (histoire), Université de Sherbrooke, 1994; pour la municipalité de Sherbrooke, T. Nootens, “'The Healthiest City in the Province of Quebec': le bureau d'hygiène de Sherbrooke face aux maladies infectieuses 1866-1911», texte manuscrit.

54. RACHPQ (1909), 50.

55. Ibid., 50. 
En 1922, le CHPQ note que généralement les municipalités acceptent «volontiers les suggestions faites par l'inspecteur régional pour l'amélioration de l'état sanitaire», mais que «dans plusieurs cas, la crise financière a été la cause d'un retard dans l'exécution de certains travaux devant améliorer la situation hygiénique ${ }^{56}{ }_{\eta}$. De telles remarques ponctuent régulièrement les rapports du CHPQ depuis leur parution en 1896. De même, les pratiques de vaccination se heurtaient à des impératifs économiques. Le CHPQ n'avait guère les moyens de distribuer gratuitement les vaccins aux municipalités et celles-ci refusaient souvent d'assumer une telle dépense. En revanche, certaines mesures préventives, telles que la déclaration des maladies infectieuses, s'avéraient peu coûteuses et faciles d'application. Elles feront pourtant l'objet de résistances farouches. Les difficultés à faire respecter la déclaration obligatoire des maladies infectieuses illustrent le défi énorme que constituait l'implantation d'une réforme sanitaire au sein des municipalités. Les données concernant la déclaration des cas en certaines régions sont à ce point lacunaires que, pour la période comprise entre les années 1888 et 1920 pour la région du Saguenay-LacSaint-Jean, les registres du CHPQ indiquent un nombre de décès causés par maladies infectieuses supérieur à celui des cas recensés ${ }^{57}$.

Les défaillances dans la chaîne de déclaration s'expliquent de plusieurs façons et reflètent la méfiance et/ou l'indifférence, voire l'hostilité de la population, des autorités municipales et même des médecins vis-à-vis certaines mesures préventives. Nombreuses étaient les familles qui évitaient de déclarer à la municipalité la présence d'une maladie contagieuse - variole, rougeole, scarlatine, etc. affectant l'un de ses membres. La population n'avait évidemment ni l'expérience ni le savoir nécessaires à l'établissement d'un diagnostic précis. Or le recours au médecin, faute de moyens financiers et en un temps où l'on acceptait parfois avec résignation la fatalité de la maladie, n'était pas toujours possible ni toujours souhaité ${ }^{58}$. Par

56. RACSHPQ (1922), 62.

57. Une telle situation n'est évidemment pas exclusive au Québec. Pour l'année 1896, seulement 25 des 57 comtés californiens avaient cumulé des statistiques vitales. Si, en 1900, presque tous les états américains avaient mis sur pied des bureaux de santé, «the majority of these boards were powerless and ineffective», mentionne J. Duffy, op. cit., 153.

58. Sur les attitudes face aux maladies infectieuses en France: J.-P. Bardet et al., Peurs et terreurs face à la contagion (Paris, Fayard, 1988); O. Faure, «De la peur aux soins: les attitudes face aux maladies épidémiques dans la région lyonnaise au XIX ${ }^{\mathrm{e}}$ siècle», Bulletin du centre d'histoire économique et sociale de la région lyonnaise (1984): 74-85; au Québec: M. Bliss, Montréal au temps du grand fléau: l'histoire de l'épidémie de 1885 (Montréal, Libre Expression, 1993). D. Goulet, Entre les miasmes..., op. cit.; D. Goulet et O. Keel, loc. cit.; G. Bilson, A Darkened House: Cholera in 19th Century Canada (Toronto, University of Toronto Press, 1980). 
ailleurs, certaines mesures sanitaires se sont heurtées à des résistances assez fortes de la part de la population et des commerçants qui s'opposaient à l'inspection, au «placardage» - panneau de $60 \times 45 \mathrm{~cm}$ avec caractères imprimés de $10 \mathrm{~cm}$ - indiquant la mise en quarantaine des domiciles infectés ou encore à la désinfection des domiciles et des établissements commerciaux. Plusieurs craignaient de se voir confinés à domicile pendant plusieurs jours ou, à l'inverse, de se faire expulser temporairement de leur logis pour en permettre la désinfection ${ }^{59}$. De plus, une famille risquait, sur ordre de la municipalité, de se voir ordonner de détruire des effets exposés à l'infection. Goulet et Keel (1991) ont montré que les mesures de désinfection, généralement bien acceptées durant les grandes épidémies, rencontraient des résistances lorsque qu'elles «étaient imposées comme une pratique routinière et récurrente ${ }^{60} \gg$.

La déclaration d'une maladie contagieuse impliquait aussi un certain nombre d'inconvénients pour les municipalités. Certains règlements du CHPQ imposaient des déboursés qui irritaient de nombreux conseils municipaux. Par exemple, les municipalités devaient défrayer les coûts occasionnés par la destruction de leurs effets personnels. À partir de 1913, les familles indigentes qui faisaient appel au médecin pour établir le diagnostic d'un malade possiblement atteint d'une maladie infectieuse n'encouraient aucuns frais, puisque les honoraires du médecin étaient à la charge des municipalités $^{61}$. Du reste, l'imposition de sanctions aux familles récalcitrantes n'étaient pas toujours prisée par les conseils municipaux qui défendaient mollement cette mesure d'hygiène publique ${ }^{62}$.

Le corps médical était aussi peu enclin à respecter la déclaration obligatoire des maladies infectieuses. Même si l'expertise des officiers hygiénistes dans le domaine de l'épidémiologie était de plus en plus reconnue par les médecins de pratique privée, notamment en situation d'épidémie, ces derniers n'ont pas toujours coopéré avec le CHPQ. Effectivement, nombreux furent ceux qui s'opposèrent à la déclaration obligatoire des maladies contagieuses en invoquant l'inviolabilité du secret professionnel. Plusieurs médecins en milieu rural étaient aussi

59. En présence d'une maladie contagieuse, le bureau d'hygiène municipal devait procéder à la déclaration des cas, à l'affichage du logis infecté, à l'isolement du malade infecté et à la désinfection «des linges du malade, de sa chambre et de son logis», $R A C H P Q$ (1895), 37.

60. D. Goulet et O. Keel, loc. cit., 216.

61. RACHPQ (1913), 6.

62. Un amendement à la loi passée en 1913 rendit plus explicite l'obligation pour une famille de déclarer la maladie et plus sévère la pénalité en cas d'omission, ibid. (1913), 6. 
opposés à la gratuité de l'immunisation et de la vaccination ainsi qu'aux mesures de désinfection obligatoire, lesquelles étaient considérées comme des empiétements inacceptables de l'État ou des pouvoirs municipaux sur leur pratique professionnelle privée.

Il faut aussi souligner que certains problèmes liés à la fiabilité du diagnostic rendaient encore plus aléatoire le bilan des maladies infectieuses déclarées. De nombreux praticiens hésitaient à recourir aux nouvelles techniques diagnostiques de laboratoire soit parce qu'ils ne disposaient pas de structures de soutien suffisantes ou encore parce qu'ils ne s'intéressaient guère aux nouveaux procédés bactériologiques $^{63}$. Ainsi, dans le cas de la diphtérie, le CHPQ déplore que «la plupart des médecins ne prennent pas la peine de faire faire d'examen au laboratoire pour le diagnostic de la diphtérie et se contentent de prendre les mesures nécessaires sans diagnostic précis ${ }^{64} \gg$. Aussi souligne-t-on que «les statistiques sur la diphtérie sont donc inévitablement très imprécises ${ }^{65}$ ». Les hygiénistes du CHPQ reprochaient à certains médecins de négliger une discrimination précise des symptômes et de confondre la variole et la varicelle ${ }^{66}$. Le CHPQ comptait améliorer la fiabilité du diagnostic en mettant sur pied en 1907 un service d'analyse de laboratoire destiné aux médecins de campagne et aux médecins en région éloignée ${ }^{67}$.

En revanche, le problème occasionné par la négligence d'un trop grand nombre de médecins en ce qui regarde la déclaration des maladies infectieuses demeure beaucoup plus difficile à solutionner ${ }^{68}$. Le secrétaire du CHPQ en est tellement excédé qu'il suggère d'exercer des poursuites judiciaires contre les médecins récalcitrants:

La suppression des maladies contagieuses souffre du fait qu'un grand nombre de médecins - peu soucieux de leurs devoirs, pour ne pas dire plus - négligent de notifier l'autorité municipale lorsqu'ils sont appelés auprès d'un contagieux. Pris en flagrant délit, ces médecins nient qu'ils ont eu à traiter une maladie contagieuse, et l'affaire en reste là. Si j'étais officier exécutif de l'autorité sanitaire dans une municipalité, voici ce que je tenterais pour mettre fin à ce stratagème coupable: je ferais une

63. Voir à ce propos, D. Goulet et $\mathrm{O}$. Keel, «Les hommes-relais de la bactériologie en territoire québécois et l'introduction de nouvelles pratiques diagnostiques et thérapeutiques (1890-1920)», Revue d'histoire de l'Amérique française, 46,3 (hiver 1993): 417-442.

64. RACHPQ (1904), 34.

65. Ibid.

66. Ibid. (1900), 65-66.

67. Ibid. (1907), 16.

68. Voir notamment: $R A C H P Q$ (1896), 12; RACSHPQ (1915), 26-27; RACSHPQ (1916), 6 et $7 ; \operatorname{RASPHPQ}$ (1924), 6; (1926), 32. 
visite à tous les médecins de la localité (dans les grandes villes une circulaire remplacerait la visite au domicile des médecins) et leur communiquerais la résolution prise par l'autorité municipale, de voir à ce que chaque cas sans exception ${ }^{69}$ soit fidèlement déclaré à l'avenir, invitant les médecins à me seconder dans mes efforts. Cela fait, je poursuivrais invariablement tout médecin pris en faute, sans m'occuper de l'issue du procès, car, traduit en cour, le médecin se verra obligé de choisir entre s'avouer coupable ou plaider l'ignorance de la médecine, c'est-à-dire l'incapacité à diagnostiquer la maladie en question ${ }^{70}$.

Rappelons que le conseil municipal, par l'intermédiaire de l'officier sanitaire exécutif du bureau d'hygiène local, avait l'obligation d'appliquer la loi d'hygiène publique et que celle-ci contenait des dispositions permettant de poursuivre les contrevenants. Mais rares furent les autorités municipales qui effectuèrent de tels recours face aux médecins exerçant dans leur juridiction. Loin d'être indépendant, l'officier sanitaire municipal - lequel était parfois médecin dépendait directement du conseil municipal et demeurait soumis aux pressions exercées sur lui. En l'absence de structure de contrôle efficace, les conseils municipaux possédaient une grande latitude dans l'application de certaines mesures de santé publique. En 1913, il n'y avait toujours qu'un seul inspecteur sanitaire pour les 1100 municipalités de la province ${ }^{71}$. Chargé d'établir une «communication avec les autorités municipales et avec les citoyens», cet inspecteur ne remplissait que partiellement son mandat et ne pouvait répondre qu'aux appels urgents des municipalités.

\section{2 - VERS UNE RÉGIONALISATION DES STRUCTURES EXÉCUTRICES DE SANTÉ PUBLIQUE: LES DISTRICTS SANITAIRES DE COMTÉ}

Se rendant compte que la politique de délégation des contrôles sanitaires aux petites municipalités était en partie un échec, le secrétaire du CHPQ prônait l'établissement d'une sorte de police sanitaire chargée de surveiller étroitement les municipalités. Au tournant de la décennie 1910, les membres du CHPQ recommandaient l'établissement de structures régionales de santé publique en territoire québécois.

Une mesure d'urgence temporaire mise sur pied en 1901 encore une fois à la suite d'une épidémie de variole - servit de

69. Souligné par l'auteur de cet extrait, E. Pelletier.

70. $R A C H P Q(1908), 14$.

71. Ibid. (1913), 28. 
modèle à cette restructuration partielle du type d'intervention sur le terrain. Pour contrer l'épidémie, les autorités gouvernementales avaient accepté, à la suite d'une recommandation du CHPQ, d'établir provisoirement dix districts sanitaires dirigés respectivement par un inspecteur. Cette mesure d'exception avait permis d'endiguer cette maladie par l'application sévère de mesures préventives (vaccination, désinfection, isolement, etc.). Même si cette initiative s'avéra fructueuse, les districts furent dissous en 1902 peu après la fin de l'épidémie. Mais l'expérience laissa des traces.

En mars 1911, à la suite d'une demande du CHPQ, le Gouvernement autorisa la division de la province en dix districts sanitaires régionaux dirigés respectivement par un inspecteur résident. En décembre de la même année, six districts sanitaires gérés par des inspecteurs régionaux diplômés furent mis sur pied: Fraserville, Québec, Sherbrooke, Montréal, Valleyfield et Trois-Rivières. Les quatre autres districts prévus - Matapédia, Saint-Hyacinthe, Hull et Chicoutimi - seront créés quelques années plus tard ${ }^{72}$.

Les officiers du CHPQ, qui avaient toujours reconnu que la compétence des officiers sanitaires constituait la pierre de touche d'une réforme sanitaire efficace, exerçaient des pressions depuis au moins 1905 afin que soient formés à l'université des officiers et des inspecteurs sanitaires compétents ${ }^{73}$. Ils obtinrent probablement gain de cause à la suite de la création des districts sanitaires puisque l'École de Médecine et de Chirurgie/Succursale de l'Université Laval à Montréal et la Faculté de médecine de l'Université Laval à Québec mirent sur pied la même année (1911) un cours d'hygiène publique appliquée. Ces cours, qui s'adressaient aux médecins et conduisaient à l'obtention du diplôme d' «hygiéniste expert», débutèrent à l'hiver $1912^{74}$.

Avec de nouveaux inspecteurs régionaux chargés d'étudier les causes de mortalité et de morbidité, de faire le relevé des maladies contagieuses et de rechercher les causes et les moyens pour en freiner

72. Le district de Chicoutimi sera opérationnel en 1917. En 1921, le conseil supérieur remaniera les districts sanitaires pour en porter le nombre à 15: Gaspésie, Rivière-du-Loup, Montmagny, Québec no 1, Québec n ${ }^{\circ}$ 2, Sherbrooke, Arthabaska, Saint-Hyacinthe, Valleyfield, Montréal $n^{\circ} 1$, Montréal $n^{\circ} 2$, Hull, Joliette, Trois-Rivières et Chicoutimi, $R A C S H P Q$ (1921), 76.

73. «Le Conseil recommande que les universités organisent et donnent un enseignement pratique spécial pour qualifier les médecins qui aspirent à devenir directeurs des services sanitaires. Le diplôme d'hygiène publique serait donc nécessaire pour exercer ces fonctions», RACHPQ (1905), 8.

74. Voir à ce propos Desrosiers et al., op. cit., et D. Goulet et A. Paradis, Trois siècles d'histoire médicale au Québec (Montréal, VLB éditeur, 1992). 
la progression ${ }^{75}$, le CHPQ comptait «pouvoir réagir effectivement contre l'inertie d'un très grand nombre de municipalités ${ }^{76} \gg$. Les nouveaux inspecteurs du CHPQ jugeront en 1914 que, parmi les municipalités faisant partie des six districts mentionnés ci-haut, «7\% ont une très bonne administration sanitaire, $19 \%$ ont une bonne administration sanitaire, $35 \%$ ont une médiocre administration sanitaire, $32 \%$ ont une insuffisante administration sanitaire, $7 \%$ sont presque dépourvues d'organisation sanitaire ${ }^{77}{ }$. Ce bilan reflète les difficultés de standardisation du contrôle sanitaire de la province et, en corollaire, les difficultés à établir des statistiques fiables en matière de maladies infectieuses. Deux ans plus tard, le CHPQ constate à nouveau que «seulement le quart des 1158 municipalités peuvent être classées comme compétentes ou du moins, bien disposées à remplir leur mis$\operatorname{sion}^{78}{ }$. Soulignons que 1031 municipalités avaient une population de moins de 2000 habitants et que la plupart de celles-ci n'avaient "pas les moyens de retenir les services de fonctionnaires compétents ${ }^{79}{ }^{\text {}}$. Le secrétaire du CHPQ misait néanmoins sur les nouveaux inspecteurs régionaux pour «surveiller de près et ramener à leurs devoirs cette immense majorité de municipalités indifférentes ${ }^{80}{ }_{\text {». }}$.

Certes avait-on assuré la formation professionnelle des inspecteurs sanitaires régionaux, mais le problème de la formation inadéquate des officiers sanitaires municipaux demeurait entier ${ }^{81}$. Certaines initiatives antérieures du CHPQ qui visaient à pallier cette lacune n'avaient guère connu de succès. De 1907 à 1911, le CHPQ avait offert sans grand succès des séances annuelles de formation pour ces officiers ${ }^{82}$.

75. Soulignons que ces inspecteurs étaient aussi tenus de déclarer toutes les maladies non infectieuses sur leur territoire.

76. RACHPQ (1914), 36. «L'œuvre accomplie par le Conseil d'hygiène pour le bien public était considérable et difficile d'exécution au début. Il fallait compter avec les obstacles toujours inhérents à l'établissement et au fonctionnement d'un service nouveau de ce genre parmi nos populations, nos populations rurales surtout, qui n'étaient préparées par aucune éducation antérieure à recevoir et à aider nos inspecteurs dans leurs travaux», ibid., 37. Sur les campagnes d'hygiène populaire, voir D. Goulet, «La théorie microbienne au service de la propagande sanitaire (1890-1920)», L'engagement social et politique des médecins, Belgique et Canada - XIXe et XXe siècles (Bruxelles, Institut Émile Vandervelde éd., 1994), 17-29.

77. Ibid. (1914), 33-34.

78. RACSHPQ (1916), 6 .

79. Ibid. (1916), 6.

80. RACHPQ (1914), 34.

81. Pour résoudre temporairement ce problème, les inspecteurs régionaux se mettaient «à la disposition des autorités municipales pour élucider certains points difficiles d'épidémiologie [et pour] rechercher [l]es sources de contagion et [l]es routes de contagion [qui dépassent] quelquefois la compétence moyenne des autorités sanitaires rurales», $R A C S H P Q$ (1916), 7.

82. Le docteur W. Johnston, bactériologiste du CHPQ, mentionne ceci en 1900: «Diplôme d'hygiène publique. - Pendant l'année dernière, une série de cours pour obtention du diplôme d'hygiène publique a été donnée dans une de nos universités et les officiers des bureaux 
Les frais de déplacement n'étant pas remboursés par leur municipalité, bien peu d'entre eux s'y inscrivirent ${ }^{83}$. En 1918, le CHPQ se plaindra encore que les conseils municipaux refusent de payer les services d'un officier de santé compétent ${ }^{84}$.

Il restait cependant un autre problème à résoudre. De nombreux médecins se montraient encore réticents à collaborer avec le CHPQ et à respecter les prescriptions des inspecteurs régionaux ${ }^{85}$. En 1918, le secrétaire du CHPQ, E. Pelletier, ne cache pas son impatience à cet égard:

La déclaration des maladies contagieuses est omise par un grand nombre de médecins, souvent même par des médecins dont la réputation n'a pas d'autres taches. Depuis que nous avons des inspecteurs régionaux, nous pouvons dire que, par eux, nous avons épuisé auprès de ces médecins négligents tous les moyens de persuasion. Il nous reste plus qu'à faire quelques exemples, et certainement que deux à quatre poursuites, dans chaque district sanitaire, auraient un effet sensible sur la profession ${ }^{86}$.

Trois ans plus tard, le CHPQ se voyait encore obligé d'adresser aux médecins une circulaire leur rappelant l'obligation légale de déclarer les cas de maladies contagieuses ${ }^{87}$. Non seulement la déclaration des maladies contagieuses était-elle lacunaire, mais au tournant des années 1920 et ce, malgré la mise sur pied d'un service

d'hygiène ont été admis gratuitement aux cours des laboratoires. Je conseille de faire connaître les avantages de ce cours aux différents bureaux locaux et de demander de faire voter les sommes nécessaires pour permettre aux officiers médicaux d'abandonner leur clientèle pendant le temps nécessaire pour suivre ces cours. Ce système a été suivi ailleurs avec grand succès. Cours pour les inspecteurs sanitaires - On a donné également des cours pour l'obtention de certificats d'Inspecteurs sanitaires, analogues à ceux du 'British Sanitary Institute'», $R A C H P Q(1900), 67$. De fait, ces cours d'hygiène mentionnés par Johnston avaient été mis sur pied à l'Université McGill en 1899. Sur les débuts de l'enseignement universitaire de la santé publique, voir G. Desrosiers, "L'introduction de l'enseignement de l'hygiène au Québec», Cahiers du Centre de recherches historiques, 12 (avril 1994): 85-104 et G. Desrosiers et al., "L'évolution des structures de l'enseignement universitaire spécialisé de santé publique au Québec 1899-1970", Bulletin canadien d'histoire de la médecine/Canadian Bulletin of Medical History (1989): 3-26.

83. $R A C H P Q$ (1911), 46

84. «Il est très difficile d'avoir un bon officier de santé pour la raison que les conseils municipaux ne veulent pas les payer, aussi l'on ne peut pas attendre beaucoup d'eux", RACSHPQ (1918), 94.

85. Pomerleau mentionne le cas d'un médecin de Knowlton qui refuse d'obtempérer aux directives sanitaires concernant la salubrité du terrain entourant sa maison. Le responsable du bureau sanitaire intervient à plusieurs reprises mais sans succès, op. cit., 115 .

86. RACSHPQ (1918), 4. Plusieurs médecins, comme en font foi les débats dans les revues médicales, considéraient cette procédure de déclaration obligatoire comme un acte détestable de délation.

87. Ibid. (1921), 11. 
de laboratoire desservant les praticiens des régions rurales, de nombreuses erreurs de diagnostic découlaient encore de la sous-utilisation des tests de laboratoire: «Les médecins [note avec irritation l'inspecteur régional du district de Québec] arriveraient facilement au diagnostic complet et précis des maladies contagieuses, s'ils avaient plus souvent recours aux procédés de laboratoire que le Conseil supérieur d'hygiène met à leur disposition ${ }^{88}$.»

Les laboratoires du Conseil d'hygiène offraient depuis le début du $\mathrm{XX}^{\mathrm{c}}$ siècle une multitude d'analyses - sang, crachat, exsudat, fèces, urine, lait, eau, etc. - aux médecins des villes et des campagnes. Plusieurs médecins avaient recours aux analyses bactériologiques et biochimiques durant les années 1920, mais c'est surtout durant la décennie 1930 que les praticiens les utiliseront sur une large échelle. Il faut dire que les laboratoires du CHPQ rencontraient certains obstacles imprévus dans la réception des échantillons. Ainsi, lorsqu'on décida d'offrir aux médecins le sérodiagnostic de la fièvre typhoïde, l'administration canadienne des postes s'objecta à la circulation par voie postale des échantillons ${ }^{89}$.

Outre les problèmes de déclaration et de diagnostic des maladies infectieuses, certains médecins cautionnaient le refus des autorités municipales de se doter d'un système adéquat d'approvisionnement en eau potable:

La profession médicale est celle qui est la mieux préparée pour aider les services publics dans ce travail éducationnel. Si chaque médecin dans sa sphère, se préoccupait de la qualité de l'approvisionnement en eau et insistait auprès des autorités locales pour obtenir les améliorations voulues, il nous serait plus facile de convaincre les municipalités de la nécessité de se protéger. Trop souvent, malheureusement, l'opposition que l'on rencontre dans les municipalités lorsqu'il faut améliorer l'eau, c'est le médecin de l'endroit qui croit que la chose n'est pas nécessaire ${ }^{90}$.

Les résistances de ces médecins, pourtant informés des méfaits d'une eau potable contaminée, s'explique en partie par les liens parfois très

88. Ibid. (1918), 72. Les laboratoires du Conseil d'hygiène offraient une multitude d'analyses - sang, crachats, exsudats, fèces, urines, lait, eau, sable, etc. - aux médecins des villes et des campagnes. Le recours à ces analyses existait déjà durant les années 1920, mais c'est surtout durant la décennie 1930 que les praticiens y auront recours sur une large échelle. Les examens pratiqués au Conseil d'hygiène entre 1928 et 1930 s'accroissent de 57232 à 80770 ; en 1940, le nombre s'élève à 285081 examens; trois ans plus tard, la division du laboratoire du CHPQ mentionne 512305 examens.

89. RACHPQ (1897), 39.

90. L'Union médicale du Canada (1928), 605. 
étroits qui les unissaient aux autorités municipales. Siégeant parfois sur le conseil municipal, leurs réticences s'accordaient à celles des municipalités quant à la déclaration des maladies infectieuses et à l'installation de certains services sanitaires jugés coûteux. Le problème n'était guère nouveau. Déjà, au tournant du $\mathrm{XX}^{\mathrm{e}}$ siècle, le CHPQ se méfiait de certains médecins et recommandait aux bureaux locaux d'hygiène de contrôler eux-mêmes la désinfection des logis après chaque cas de maladie contagieuse en suivant une méthode prescrite: «Le Conseil ne fait pas confiance aux médecins dans ce cas, car il ne s'agit pas de protéger un malade mais ceux qui l'entourent et qui sont en santé91.»

Que ce soit par refus de déroger au secret professionnel en ce qui regarde la déclaration des maladies infectieuses ou encore par simple négligence, les médecins délinquants nuisaient considérablement au succès des entreprises de réformes sanitaires du CHPQ. Élément central des soins de santé dans un système professionnel libéral axé sur l'offre et la demande, le médecin possédait un pouvoir de persuasion important, particulièrement dans les petites communautés rurales du Québec. En revanche, son refus d'obtempérer à certaines mesures d'hygiène publique minait la crédibilité des mesures adoptées par le CHPQ. Le docteur A. Lessard en fait le dur constat:

Le nombre de médecins qui ne déclarent pas les cas de maladies contagieuses est encore trop grand. Je me demande si réellement ceux qui manquent au devoir que leur commande la loi, ont bien le sens de leur responsabilité. Cacher aux autorités un cas de diphtérie, de scarlatine, de rougeole, de coqueluche, etc., c'est d'abord donner un triste exemple à ses concitoyens et surtout c'est donner aux autorités municipales l'impression que la Loi et les Règlements provinciaux d'hygiène sont choses de très minime importance, puisque ceux qui devraient être les premiers à les observer sont les premiers à les enfreindre ${ }^{92}$.

La création de districts sanitaires et la nomination d'inspecteurs hygiénistes dans ces districts avaient certes pour but de sensibiliser plus efficacement certaines municipalités aux mesures prophylactiques et de susciter une meilleure collaboration des intervenants en faisant appel au sens civique du citoyen et à la conscience professionnelle des autorités municipales et médicales. En réalité, les inspecteurs régionaux se sont retrouvés dans l'inconfortable situation d'exercer une

91. RACHPQ (1896), 15.

92. RASPHPQ (1924), 6. 
sorte de police médicale ${ }^{93}$ dans la plus pure tradition de la médecine préventive du $\mathrm{XIX}^{\mathrm{e}}$ siècle: «Soixante-quinze pour cent des municipalités de la province ne font du travail sérieux que lorsqu'elles sentent la surveillance des officiers du Conseil d'hygiène et même, il faut assez souvent que l'officier provincial stationne dans l'endroit pour que les mesures qu'il a fait prendre soient maintenues ${ }^{94}$.» Il devint de plus en plus évident qu'il fallait modifier les sphères de responsabilité en faveur d'un interventionnisme accru du CHPQ et d'un encadrement plus serré des petites municipalités par des hygiénistes de carrière.

\section{3 - UNE NOUVELLE STRUCTURE RÉGIONALE EXÉCUTRICE: LA CRÉATION DES UNITÉS SANITAIRES DE COMTÉ}

Constatant au début des années 1920 «que la province ne peut suppléer au manque d'organisation compétente dans les régions rurales en augmentant indéfiniment le nombre de ses inspecteurs régionaux ${ }^{95}$ ", le CHPQ en était venu à la conclusion qu'il fallait remplacer les bureaux de santé municipaux dans les petites localités par une organisation sanitaire de comté regroupant plusieurs municipalités et dirigée par un personnel spécialisé. Déjà exposé en 1913 par le secrétaire du CHPQ qui souhaitait prendre comme unité administrative le comté, ce projet de regroupement des structures sanitaires en milieu rural prit naissance dans la foulée d'initiatives similaires prises en Angleterre, en France, aux États-Unis ainsi qu'au NouveauBrunswick $^{96}$.

Il s'agissait en quelque sorte de délester les municipalités de certaines responsabilités en matière de santé publique (vaccination, hygiène privée, soins infantiles, etc.) au profit des structures régionales de contrôle. Aussi demanda-t-on au pouvoir législatif la modification de la Loi d'hygiène publique afin que soient créés des secteurs d'intervention - encadrés par des hygiénistes compétents -

93. L'expression «police sanitaire» s'appliquait parfois au sens propre puisque des policiers faisaient parfois office d'inspecteurs des mesures d'hygiène. À la Nouvelle-Orléans, dès les premières manifestations d'une épidémie, les policiers recevaient «l'autorité nécessaire pour enquêter sur les conditions sanitaires de toute maison ou bâtiment de la ville», G. Vandal, loc. cit., 11 .

94. RACSHPQ (1916), 7.

95. Ibid. (1921), 71.

96. «L'État de l'Ohio a jeté par-dessus bord tous les petits 'Local Boards of Health' incompétents pour réunir, sous une même officialité, un territoire comprenant plusieurs municipalités [...]. Une des provinces du Canada, le Nouveau-Brunswick, a des bureaux d'hygiène de comté et croit à la supériorité de cette organisation sanitaire de comté, sur les petites organisations municipales, comme celles que nous avons dans le Québec», ibid., 70-71. 
qui regrouperaient plusieurs municipalités. Le modèle proposé s'inspirait largement de la structure américaine de santé publique financée par le Bureau international de santé de la fondation Rockefeller. Le directeur du CHPQ, accompagné de deux officiers sanitaires, avait effectué un séjour aux États-Unis en avril 1925 afin de s'informer des organisations de santé publique mises en place dans les villes, les états et les comtés américains ${ }^{97}$.

À la suite de la loi amendant la Loi d'hygiène publique votée en 1926, des unités sanitaires, sous la direction du docteur A. Lessard, sont mises sur pied à titre d'essai par le CHPQ dans trois comtés ruraux de la province: Beauce, Saint-Jean d'Iberville et Lac Saint$\mathrm{Jean}^{98}$. Ces unités financées conjointement par le CHPQ, par la fondation Rockefeller et par les municipalités étaient composées d'un médecin hygiéniste à temps plein, de deux ou plusieurs infirmières visiteuses, d'un inspecteur sanitaire «chargé de la police sanitaire et de l'éducation des officiers municipaux ${ }^{99}{ }^{\gg}$ et d'une secrétaire. Lors d'un congrès tenu l'année suivante, l'Union des municipalités reconnaîtra les services rendus par ces unités et adoptera une motion pour en recommander la multiplication. En 1930, il y aura 23 unités sanitaires établies dans la province.

La création de ce nouveau palier d'intervention signifiait à toutes fins utiles un transfert de responsabilité du niveau municipal au niveau régional en matière de santé publique. Les tâches d'éducation, de dépistage et de prévention seront largement assumées et/ou supervisées par les officiers des unités sanitaires. Les fonctions de surveillance et de contrôle y sont aussi manifestes. Le principal instigateur de cette réforme, le docteur A. Lessard, directeur du CHPQ, exprimait le souhait que grâce aux unités sanitaires, «le territoire et la population [du comté] se trouve[ro]nt ainsi sous une surveillance constante» et que «pas une municipalité n'échappe[ra] à

97. La fondation Rockefeller avait aussi octroyé en 1925 des bourses d'études à quatre fonctionnaires du CHPQ: un médecin étudiait l'épidémiologie à Johns Hopkins; un bactériologiste et un ingénieur sanitaire, à Harvard et une infirmière, à Columbia, $R A S P H P Q(1926)$, 14.

98. SPQ (1926), «Loi modifiant la loi de l'hygiène publique du Québec», chap. 54. Sur la création des unités sanitaires de santé, voir G. Desrosiers et al., «Vers un système de santé publique au Québec. Histoire des unités sanitaires de comté: 1926-1975», Rapport de recherche (département d'histoire et département de médecine préventive, Université de Montréal, 1990); D. Goulet et A. Paradis, op. cit., 289-290, 293-296, 298-299.

99. RASPHPQ (1926), 14. À propos du financement tripartite de ces unités et du rôle joué par la fondation Rockefeller, voir ibid., 16. La fondation Rockefeller, inaugurée en 1913, avait pour mission de promouvoir la santé publique à travers le monde et elle avait coopéré peu après sa création à l'organisation de services d'hygiène en Amérique latine, en Australie, en Chine et avait mis sur pied des campagnes antituberculeuses aux États-Unis et en France. 
l'observation des officiers ${ }^{100}{ }_{»}$. Les officiers municipaux seront désormais fortement encadrés par l'inspecteur sanitaire qui effectuera les pressions nécessaires afin que soient respectées, dans les limites des possibilités financières des municipalités, les dispositions de la loi d'hygiène publique concernant l'ingénierie sanitaire: adduction d'eau, égouts, ébouage, etc. ${ }^{101}$. Cette nouvelle structure de santé publique basée sur un contrôle régional et dotée d'un personnel spécialisé à temps plein posait les premiers jalons d'une politique de prévention et d'éducation systématique couvrant l'ensemble du territoire québécois et contribuera à rendre plus efficaces les procédures de déclaration et d'éradication des maladies infectieuses.

\section{CONCLUSION}

L'organisation d'une structure d'hygiène à l'échelle provinciale, basée sur la collaboration plus ou moins volontaire des autorités municipales durant les premières décennies du $X X^{\mathrm{e}}$ siècle, a certes contribué à améliorer les conditions sanitaires des municipalités québécoises. Les interventions du CHPQ se sont généralement inscrites dans la lignée d'une médecine préventive conservatrice axée sur les études épidémiologiques et l'ingénierie sanitaire. Si une telle orientation s'est faite au détriment d'une approche sociale plus globale des problèmes de santé, elle a néanmoins permis d'instituer des mesures de contrôle de longue durée et de contrer les résistances de nombreux représentants municipaux qui préconisaient plutôt l'application transitoire et ponctuelle des mesures d'hygiène. Il ressort de notre étude que l'amélioration de la santé publique basée sur une politique de collaboration volontaire des autorités municipales s'est manifestée de façon très inégale sur le territoire québécois et peut être considérée, surtout en milieu rural, comme un demi échec.

L'absence de collaboration de certaines municipalités au projet sanitaire, l'appui timide de nombreux médecins et la formation inadéquate des responsables municipaux en cette matière rendaient inopérantes certaines dispositions de la loi d'hygiène publique. Les membres du CHPQ ne possédaient, jusqu'à la fondation des unités sanitaires de comté, que des moyens limités pour appliquer leur réforme sanitaire. Certes, les contingences administratives et financières ont aussi joué un rôle important qu'il faudrait analyser en profondeur, mais elles n'expliquent pas tout. D'autres facteurs ont aussi joué: absence de structures de contrôle efficace, manque de per-

100. Ibid., 14.

101. Ibid. 
sonnel à temps plein spécialisé en hygiène publique (médecins, infirmières, secrétaires), indifférence de certaines autorités locales, résistance de la population à certaines mesures jugées coercitives, méconnaissance des progrès prophylactiques et étiologiques, difficulté de normalisation, etc. Il demeure que les activités du CHPQ créature de l'État - entre 1886 et 1926 ont largement contribué, comme le soulignait Guérard ${ }^{102}$, au processus de médicalisation de la société québécoise. Ce processus aura d'ailleurs tendance à s'accélérer avec l'entrée en scène des unités sanitaires de comté. Issues d'une volonté d'accroître la régionalisation des instances exécutrices au détriment des municipalités, d'assurer l'uniformisation des mesures sanitaires et la spécialisation du personnel, elles modifieront largement l'approche structurelle de la santé publique en y ajoutant notamment une dimension sociale jusqu'alors peu présente dans les enjeux médicaux. 